\title{
Guest editorial: Special issue on genetic programming, evolutionary computation and visualization
}

\author{
Nadia Boukhelifa ${ }^{1} \cdot$ Evelyne Lutton $^{1}$ \\ Published online: 11 August 2018 \\ (c) Springer Science+Business Media, LLC, part of Springer Nature 2018
}

Recent advances in genetic programming/evolutionary computation (GP/EC) and visualization have shown a growing synergy between these fields. For example, on the one hand, visualization can make GP/EC algorithms more transparent, through interactive parameter tuning and the graphical representation of experimental analyses. On the other hand, GP/EC techniques can be deployed to efficiently guide user search in exploratory visualization systems. The goal of this special issue was to bring together latest work and new advances in genetic programming/evolutionary computation (GP/EC) and visualization.

Following an open call for papers disseminated in the GP/EC community as well as through visualization (Vis) and human-computer interaction (HCI) mailing lists, we received 16 submissions. Reviewers from the two domains (GP/EC and Vis/ $\mathrm{HCI}$ ) were assigned to each paper, to make sure all aspects of the work are evaluated in depth. In the end, five papers were accepted for publication. We note that all submissions to this special issue came from the GP/EC domain. As part of the revision process, authors of conditionally accepted papers were asked to extend their discussions to cover the visualization design choices and to describe supported tasks in reference to established information visualization taxonomies. They were also asked to provide proof of validation with target user groups, even if these were preliminary, whether in the form of use cases, questionnaires or user studies. Finally, they were asked to include research perspectives such as the top visualization challenges that need to be addressed as future work.

The first three contributions of this special issue show how visualisation can be used to improve the understanding of the behaviour of evolutionary algorithms:

- For general purposes: "Visualising the Global Structure of Search Landscapes: Genetic Improvement as a Case Study" by Nadarajen Veerapen and Gabri-

Nadia Boukhelifa

nadia.boukhelifa@inra.fr

Evelyne Lutton

evelyne.lutton@inra.fr

1 INRA, Université Paris-Saclay, 1 av. Brétignières, 78850 Thiverval-Grignon, France 
ela Ochoa presents a landscape analysis based on local optima networks, and "Unveiling Evolutionary Algorithm Representation with DU Maps" by Eric Medvet, Marco Virgolin, Mauro Castelli, Peter A. N. Bosman, Ivo Gonçalves, and Tea Tušar deals with the difficult issue of analysing the representation of solutions.

- For cooperative co-evolution in the context of a medical imaging application: "Data exploration in evolutionary reconstruction of PET images" by Cameron C. Gray, Shatha F. Al-Maliki and Franck P. Vidal.

The two remaining papers are focussed on advanced and adapted visualisations of the algorithmic outputs for various evolutionary schemes:

- For many-objective optimisation: "Visualisation with Treemaps and Sunbursts in Many-objective Optimisation" by David J. Walker proposes a Pareto front visualisation with special attention to diversity and convergence.

- For evolutionary learning: "VALIS: an Evolutionary Classification Algorithm" by Peter Karpov, Giovanni Squillero and Alberto Tonda shows how the structure of the algorithm allows a convenient animated visualisation.

Some of the proposed approaches are offline, i.e. data are analysed a posteriori, after the algorithm runs, like the work by Veerapen et al. and Cameron et al. Others are offline, but are readily extendable to online visualisation, i.e. the progressive display of population data along the algorithmic run, like the work by Walker and Karpov et al.

From the visualization point of view, all accepted papers handled abstract datasets such as the population runs of EC/GP algorithms and their diversity and usage, the structure of computational search spaces, and the process of evolutionary classification. Established information visualization techniques were deployed including trees and networks, sunburst diagrams, heatmaps, scatterplots and parallel coordinate plots. These visualization techniques were used mostly for representation and communication purposes, and less for interactive analysis or exploration. The novelty of the proposed methods, however, lied in applying such techniques to interesting and challenging EC/GP problems. This often required considerable efforts, first to articulate and transform abstract problems into visual or geometrical representations, and then to evaluate these methods and validate the findings.

As guest editors of this special issue, we carefully examined the feedback and discussions provided by the authors and reviewers throughout the revision cycle. This allowed us to distill a number of high level observations pertaining to the visualization design choices, validation and challenges. For example, it was interesting to see authors motivate the usage of visualization to solve their respective GP/EC problems through general descriptors of visualization as 'natural' or 'intuitive', stating that it makes their algorithms more 'transparent', 'accessible' and 'understandable' to users. These types of comments perhaps arise because for all the papers in this issue the intended user groups are the EG/EC practitioners themselves (with the exception of work by Walker which also targets problem owners). These claims are hard to verify without formal validations, but they tend to reflect the willingness of 
the EG/EC community to explore how visualization techniques can be used to tackle difficult research problems.

Having said that, we were delighted to see that in many cases, the validation of the contributions went beyond rigorous algorithmic and experimental analysis. Although most of the results provided in terms of user-centered evaluations are preliminary and have limitations, different evaluation methods were used: one controlled lab experiment, one online user study, and one questionnaire. This is indeed very encouraging as we strongly believe that in systems where humans and algorithms collaborate to solve a problem, both algorithm-centered and user-centered evaluations are needed. However, in their letters to reviewers, many authors indicated user evaluation as a key challenge. Understandably, EC/GP practitioners do not necessarily have formal training on user evaluation methodologies. This calls for close collaboration between $\mathrm{HCI} / \mathrm{V}$ is and GP/EC practitioners, in particular to help evaluate the interplay and co-adaptation between algorithms and users.

Other challenges in applying visualization to EC/GP problems raised by the authors of this special issue include the handling of high-dimensional data, visual clutter, supporting interactivity and the progressive rendering of algorithmic results, and the integration of visualization and EC/GP algorithms. The latter issue is not a trivial one. A number of authors highlighted the importance of making visualization tools more easily integratable to existing analysis and computational platforms used by the EC/GP community.

There exists a fertile ground for innovative research at the intersection between EC/GP and visualisation. The five papers of this special issue demonstrate how visualization can improve the understanding of the behaviour of evolutionary algorithms and their output. More work is needed to address the visualization challenges such as those discussed in this special issue.

The guest editors express their appreciation to the authors for their high quality work and their contribution to the state of the art of this emerging domain. We would like to thank all reviewers for their helpful feedback and comments. We thank Professor Lee Spector for initiating this special issue, and the Springer team for their support.

We hope you find this special issue both interesting and inspiring.

Publisher's Note Springer Nature remains neutral with regard to jurisdictional claims in published maps and institutional affiliations. 